\title{
LA EVALUACIÓN DE PROGRAMAS E INTERVENCIONES SANITARIAS EN EL MARCO DE LOS PROGRAMAS PRESUPUESTALES DE SALUD
}

\author{
THE EVALUATION OF HEALTH PROGRAMS AND INTERVENTIONS WITHIN THE FRAMEWORK \\ OF BUDGETARY HEALTH PROGRAMS
}

\author{
Alfonso Gutiérrez-Aguado $1,2, a, b, c$
}

Año a año, la asignación de recursos en salud se ha incrementado. Para el año 2009 fue de S/ 6,9 mil millones y en el año 2017 asciende a S/ 17,2 mil millones, este incremento debe ir a la par con la mejora de resultados sanitarios; esta forma diferente de asignar recursos y buscar resultados es lo que se denomina presupuesto por resultados (PpR), el cual viene implementándose desde el 2007.

EIPpR tiene cuatro instrumentos que permiten su operatividad, el primero de ellos son los programas presupuestales. Cabe precisar que el diseño de un programa presupuestal está basado en evidencias, contribuyéndose en una gran oportunidad para los investigadores, ya que sus hallazgos permiten identificar factores causales de los principales problemas sanitarios. Así mismo, permite identificar a través de evidencia científica, las intervenciones más efectivas para el logro de resultados. A la fecha, se cuenta con nueve programas presupuestales que abordan los principales problemas de salud pública (desnutrición, mortalidad materna y neonatal, tuberculosis, VIH/SIDA, enfermedades metaxénicas y zoonóticas, hipertensión arterial, diabetes mellitus, salud bucal, salud ocular, intoxicación por metales pesados, cáncer, problemas de personas con discapacidad, emergencias y urgencias médicas y salud mental).

El segundo instrumento es el seguimiento o monitoreo de indicadores, lo cual implica reunir datos de diversas fuentes, identificar un conjunto de indicadores y metas para aportar información oportuna a los diferentes niveles de gobierno. El tercer instrumento son las evaluaciones independientes, las cuales se realizan sobre la base del monitoreo, con un análisis más profundo que tiene en cuenta los cambios contextuales, aborda asuntos relativos a la atribución y analiza en muchos casos situaciones hipotéticas. El cuarto y último instrumento son los incentivos a la gestión, por medio de los cuales los gobiernos locales y regionales se involucran en el cumplimiento de metas ${ }^{(1)}$.

Los programas presupuestales en salud están estructurados sobre una cadena de valor (insumos, actividades, productos y resultados). En cada eslabón de esta cadena se pueden identificar indicadores que, posteriormente, se vinculan con métodos de recopilación de datos. Este modelo de cadena de resultados permite enfatizar las necesidades de análisis, pero, sobre todo, permite monitorear y luego evaluar las intervenciones que forman parte de un programa.

La evaluación se convierte en una herramienta clave en este proceso, pero bajo esta estructura y en la interacción con el sector Salud, nos encontramos con una serie de problemas para desarrollar evaluaciones de los programas e intervenciones sanitarias, tales como no contar con línea de base de los indicadores y que las metas se establecen solo para algunos indicadores, los evaluadores tienen problemas de accesibilidad a los datos, los análisis de los programas se llevan a cabo por separado y sin referencias o vínculos con contexto del sector Salud.

Normativa y estructuralmente el Ministerio de Salud (MINSA) (Ley General de Salud y Reglamento de Organización y funciones) hace referencia sobre la importancia de evaluar las direcciones de línea de la Dirección General de Interverciones Estratégicas, las cuales tienen la función de realizar acciones de monitoreo, seguimiento y evaluación, en coordinación con los actores del sector Salud y otros sectores del Estado y la sociedad civil, a fin de mejorar o rediseñar las intervenciones estratégicas.

¿Qué deberíamos hacer?, desarrollar competencias en los recursos humanos, específicamente capacidades en aspectos de evaluación de programas e intervenciones sanitarias, contar con una institución que se dedique a evaluar las intervenciones sanitarias, desde su diseño, sus procesos, impacto, pero, sobre todo, si son costoefectivos (evaluación de tecnologías sanitarias).

\footnotetext{
Ministerio de Economía y Finanzas. Lima, Perú.

Universidad Nacional Mayor de San Marcos. Lima, Perú.

Editor invitado, ${ }^{\mathrm{b}}$ médico especialista en Gestión en Salud, ${ }^{\mathrm{c}}$ magíster en Salud Pública

Recibido: 11/09/2017 Aprobado: 13/09/2017 En línea: 29/09/2017
}

Citar como: Gutierrez-Aguado A. La evaluación de programas e intervenciones sanitarias en el marco de los programas presupuestales de salud. Rev Peru Med Exp Salud Publica. 2017;34(3):363-4. doi: 10.17843/rpmesp.2017.343.3139 
Así mismo, deberíamos mapear, sistematizar y publicar la data de monitoreo y seguimiento existente en el sector. Los sectores acumulan gran cantidad de información que puede ser útil para el avance del conocimiento científico y la innovación en la política pública

Además de tener un sistema de información, regular, oportuno y accesible sobre la evolución de los indicadores y los resultados alcanzados de los programas y políticas de salud, desarrollar alertas periódicas y recomendaciones para la toma de decisiones respecto si estamos marchando bien o es necesario hacer ajustes en las intervenciones de las políticas de salud, teniendo en cuenta las nuevas evidencias nacionales e internacionales y los escenarios locales, regionales, nacionales.

Así también, incentivar la participación de las organizaciones de la sociedad civil en el diseño y evaluación de las políticas de salud, mediante reuniones de trabajo que tengan como objetivo la producción de un balance de los resultados logrados, desde un enfoque de derechos y cierre de brechas. Un ejemplo exitoso se ha dado a través de los grupos de trabajo de las mesas de concertación para la lucha contra la pobreza, en la que participan actores del Estado y sociedad civil.

Un actor clave en este proceso de evaluación es la participación de la academia nacional en el diseño y evaluación de políticas de salud, mediante la generación de "esquemas de aprendizaje" en los que investigadores y diseñadores de política generan modelos de intervención innovadores que son acompañadas de evaluaciones de impacto experimentales.

Finalmente, la evidencia producida a través de estas evaluaciones debe ser considerada un bien público, y como tal debe ser publicada y difundida para el avance del conocimiento científico así como de la política pública nacional e internacional. Una propuesta hacia el futuro es que las evaluaciones en el marco del PpR busquen no solo mejorar el gasto público mediante la verificación de productos y resultados, sino probar hipótesis relevantes para la ciencia y la política pública mediante la evaluación de estrategias piloto, que potencialmente puedan mejorar los resultados del programa presupuestal mediante la innovación de alguno de sus componentes.

En este número de la Revista se presenta el simposio "Evaluación de Programas e Intervenciones sanitarias"; en concordancia con el objetivo de este simposio, se ha invitado a diferentes especialistas en la materia: un médico epidemiólogo responsable de monitoreo y evaluación del MIDIS, y economistas coordinadores de seguimiento y evaluación en el marco de los programas presupuestales del Ministerio de Economía y Finanzas.

El estudio titulado "Los efectos de los programas sociales en la salud de la población en condición de pobreza: Evidencias a partir de las evaluaciones de impacto del Presupuesto por Resultados a Cuna Más, Juntos y Pensión 65 " (3), tiene como finalidad describir la experiencia en la gestión de evaluaciones de impacto del MEF como uno de los instrumentos del PpR y documentar el diseño y resultados de dichas evaluaciones sobre sus resultados principales y sobre indicadores que miden el acceso a los servicios de salud y el estado de salud de su población objetivo, en relación a la salud materno infantil y la salud emocional y física del adulto mayor, tema presentado por las economistas Viviana Cruzado, Denice Cavero y Gabriela Cuadra.

Las economistas Karina Peñaloza, María Claudia Prado y el magíster Alfonso Gutiérrez en el artículo que presentan sobre "Evaluaciones de diseño y ejecución presupuestal, un instrumento del presupuesto por resultados: algunas experiencias aplicadas en salud" (4) presenta las principales características del proceso de las Evaluaciones de desempeño y ejecución presupuestal (EDEP), sus métodos y principales herramientas de evaluación, la matriz de preguntas de evaluación y la definición de la matriz así como el seguimiento a sus compromisos de mejora del desempeño a partir de las recomendaciones de las EDEP. Se concluye mostrando su aplicación a intervenciones públicas en salud.

Finalmente, con el objetivo de monitorear y evaluar los programas presupuestales el MINSA desarrolló, con el Instituto Nacional de Estadística e Informática, seis encuestas poblacionales: Encuesta Nacional de Hogares, Encuesta de Demografía y Salud Familiar, Encuesta de Programas Presupuestales, Encuesta de Establecimientos de Salud que mide Funciones obstétricas neonatales, Encuesta de Crecimiento y Desarrollo y la Encuesta de Riesgo Anual de Infección a Tuberculosis. Su importancia en evaluación de programas se presenta en el artículo "Encuestas en Salud: Instrumentos esenciales en el seguimiento y evaluación de los Programas Presupuestales", presentado por la economista Rosmery Rivera y el magíster José Enrique Velásquez ${ }^{(5)}$.

\section{REFERENCIAS BIBLIOGRÁFICAS}

1. Perú, Ministerio de Economía y Finanzas (MEF). Programas presupuestales. Diseño, revisión y articulación territorial, 2016 [Internet]. Lima: MEF; 2016 [citado el 15 de septiembre 2017]. Disponible en: https://www.mef.gob.pe/contenidos/ presu_publ/ppr/directiva_PP2016.pdf

2. Issel M, Wells R. Health program planning and evaluation, a practical, systematic approach for community health. 4a ed. Burlington, MA: Jones \& Bartlett Learning; 2017.

3. Cruzado V. Los efectos de los programas sociales en la salud de la población en condición de pobreza: evidencias a partir de las evaluaciones de impacto del presupuesto por resultados a programas sociales en Perú. Rev Peru Med Exp Salud Pública.2017;34(3):528-37.

4. Peñaloza K, Gutiérrez-Aguado A Prado M. Evaluaciones de diseño y ejecución presupuestal, un instrumento del presupuesto por resultados: algunas experiencias aplicadas en salud. Rev Peru MedExpSaludPública.2017;34(3):521-7.

5. Velásquez-Hurtado JE, Rivera-Sivirich RA. Encuestas en salud: instrumentos esenciales en el seguimiento y evaluación de los programas presupuestales. Rev Peru Med Exp Salud Publica. 2017;34(3):512-20. 